\title{
Optimal Districting with Endogenous Party Platforms
}

\author{
Emanuele Bracco
}

\begin{abstract}
Representation is one of the most important criteria by which to judge electoral systems. In this paper, I focus on one aspect of representative democracy: the formation of electoral district boundaries. It is well known that majoritarian systems give rise to highly biased seat-vote curves, causing representation to be less than ideal. What should, therefore, be the optimal constituency design when the objective is to maximize voters' welfare? I show that when parties take account of districting while setting platforms, then the district design problem reduces to a very simple rule: do nothing when voters are risk neutral, and - when voters are risk averse - choose a bias that is against the largest partisan group. Calibrating the model on data of U.S. State legislative elections during the 1990s, I show that the welfare gain due to optimal districting is very small.

JEL Classification: D70, D72, H7
\end{abstract}

Keywords:

Districting, seat-vote curve, social planner, policy-motivated parties, office-motivated parties, voting, legislative elections

Email address: e.bracco@lancaster.ac.uk. Department of Economics, Lancaster University, Lancaster, LA1 4YW, UK. Telephone: +44 (0) 1524592728 . Fax: +44 (0) 1524594244 (Emanuele Bracco)

URL: http://www.lancs.ac.uk/staff/bracco/ (Emanuele Bracco) 


\section{Introduction}

One of the major distortions in the electoral process comes from the move from direct to representative democracy, i.e. the way popular votes are transformed into policies through representation. One of the most famous is the Electoral College system, which is used to elect the U.S. President. It is well known that George W. Bush won the 2000 U.S. Presidential elections receiving fewer popular votes than his rival Al Gore. In countries which elect the legislature through single-member districts, the way district boundaries are designed is of paramount importance. In Britain, for example, the electoral constituencies are such that the Labour party enjoys a bias in its favor; it has been calculated that the Conservative Party may need up to a $10 \%$ lead in popular votes to obtain a parliamentary majority.

The distortionary effects of districting have been studied extensively in recent years. In particular, Coate and Knight (2007) were the first to perform a complete normative analysis of districting. Their paper, however, did not consider how parties' policy choice is affected by the very districting, and assumed that parties platforms are exogenously fixed. This paper takes into account that parties choose their policy platform taking into consideration the disortionary effects of the districting, and claims that a normative analysis on the social welfare effect of districting ought to consider this feature.

I develop a model based on Coate and Knight (2007) and Besley and Preston (2007), which allows for parties to choose their policy platform after the district boundaries have been designed. This timing is very realistic, especially considering how several elections are held while holding the district boundaries constant. In the U.S., for example, redistricting is performed by law every ten years, in correspondence with each Census; therefore five consecutive elections are held before the boundaries are revised. Moreover, in many countries - 44 out of 60 countries surveyed by Handley (2008) but, notably, not the U.S. - the revision of district boundaries is performed by independent commissions. These include countries like the UK, Australia and Canada. Among nations with single-member districts, only U.S. and French legislators have substantial powers in the redistricting process.

In the U.S. redistricting is highly contentious and explicitly in the hands of state legislatures and governors. Some U.S. states have devolved the power of redistricting to independent or bipartisan commissions, with the nominal aim of reducing the partisanship of this important aspect of electoral democracy. At the moment only nine states have appointed a special commission for this task, ${ }^{1}$ thirteen more use a mixed commissionbased legislative-based process in which the political actors still hold some power. In the majority of states the re-districting is still fully in the hands of politicians. Even when redistricting is kept at arm's length from party politics, it is not always clear what are the criteria that an independent districting commissions should follow.

Operating this change - i.e. allowing parties to endogenously set their platforms conditional on the districting-, conventional wisdom is overturned, in particular with respect to the seat-vote curve. Anecdotal knowledge would suggest to aim at an unbiased seatvote curve, such that if each party gains $50 \%$ of the votes, each one is awarded exactly

\footnotetext{
${ }^{1}$ Alaska, Arizona, California, Hawaii, Idaho, Missouri, Montana, New Jersey, and Washington.
} 
$50 \%$ of the seats. Coate and Knight (2007) stated that the socially optimal seat-vote curve should be (weakly) biased in favour of the most represented party in the population. My results demonstrate instead that the social planner and parties' equilibrium strategies are compatible with an optimal seat-vote curve, which is biased in the opposite direction with respect to Coate and Knight's (2007) finding: a weakly negative bias, that counterbalances the weight of the larger partisan group.

The intuition of this result can be easily explained. Each party's Nash equilibrium strategy (i.e. policy platform) is to "follow" the districting ideological leaning, in order to maximise the chances of victory: proposing more leftist (rightist) platforms, if the districting is favoring the Democrats (Republicans). The social planner, then, needs to design a median district that mirrors the population's ideological composition, so that both parties' policy platforms are endogenously dragged towards the social optimum. This cancels the need to artificially increase the electoral weight of the votes obtained by the larger party, leading to an electoral result in which the majoritarian party wins a small majority of seats with a large(r) majority of votes.

This prediction is brought to the data from 28 U.S. State Lower-House elections throughout the Nineties. ${ }^{2}$ This exercise underlines how the welfare gain from implementing the socially optimal districting would be very small: under endogenous party platform, parties move their policies towards the center irrespectively of the districting, in search of the Independents' vote; the difference in policies between actual and optimal districting is therefore quite small, and so is the overall welfare gain.

In addition to the papers already cited, the current paper is related to several other literatures. In particular, other works analyzed the welfare effects of districting, albeit with a narrower focus: Hinich and Ordeshook (1974) look into the Presidential electoral system in the U.S. to see whether the Electoral College mechanism favors voters of small or large states, focusing as well on the distance between the median voter and the median elected representative (i.e. the median member of the Electoral College). Similarly to this paper, they arrive at the conclusion that one needs to look at the pivotal (i.e. median) member of the relevant assembly: the Electoral college for US Presidential elections, or the State legislatures for this paper. Gilligan and Matsusaka (2006) looked into the effects of gerrymandering on the distance between the median voter's preferences and those of the median representative. Shotts (2002) and Epstein and O'Hallaran (2004) focus instead on minorities, analyzing the effects of majority-minority districts on their welfare. Gul and Pesendorfer (2010) looks into how gerrymandering affects policies at the federal level, i.e. in a situation in which each party has the power of redistricting only a share of the electoral districts.

This work also contributes to the literature on electoral competition. In particular my results can be easily related to those obtained by Wittman (1983), Calvert (1985), Callander (2008), and Bernhardt et al. (2009). All of them move forward from the policy convergence result of Downs (1957), assuming - as it is in this paper - simultaneously

\footnotetext{
${ }^{2}$ The dataset, compiled by Ansolabehere and Snyder (2002), has been kindly provided by Brian Knight, to whom we are greatly indebted. This dataset was used for the empirical analysis in Coate and Knight (2007).
} 
office- and policy-motivated parties; the latter in particular focuses on how policy divergence affects social welfare.

More generally, this paper relates with the wider literature on the optimal design of political institution: Lizzeri and Persico (2001) focused on electoral systems, Lockwood (2002) on the distribution of tasks across tiers of government, Prat (2002) and Coate (2004) on campaign finance, Persson et al. (2000) on the pros and cons of parliamentary rather than presidential systems. All of these papers overlook the effects of districting on electoral outcomes and welfare.

This paper's empirical analysis can be related to the long-standing literature which tries to estimate the seat-vote curve. The seminal paper of this literature is Kendall and Stewart (1950), which used British data and a log-odds functional form, and stated the famous "Cube Law," referring to the responsiveness of the British electoral system. Gelman and King (1994), among others, developed a statistical methodology to estimate the seat-vote curve and to predict the effect of majority-minority districts.

The rest of the paper is organized as follows: Section 2 describes the theoretical model, in Section 3 the model is solved, Section 4 contains the econometric analysis, and Section 5 concludes.

\section{The Model}

There exists a continuum of voters of mass 1 with Euclidean preferences defined over the policy space $[0,1]$. Letting $i$ be the bliss point of a given voter, and $x$ the implemented policy, her utility is defined as:

$$
u_{i}=L(i, x)
$$

where $L(i, x)=-|i-x|^{\rho}$ and $\rho \geq 1$ determines the degree of convexity of voters' preferences.

Voters are characterized by their preferred policy, and are of three types: Democrats, Republicans, and Independents, of mass respectively $\bar{\pi}_{D}, \bar{\pi}_{R}$, and $\bar{\pi}_{I}$, with $\bar{\pi}_{D}+\bar{\pi}_{R}+\bar{\pi}_{I}=$ 1. The Democrats' ideal policy is 0 and the Republicans' is 1 . We restrict our attention to cases in which no type has an abolute majority, and there is at least $15 \%$ of the population belonging to each type $\left(\bar{\pi}_{s} \in(.15, .5)\right.$, for $\left.s \in\{D, R, I\}\right) .^{3}$

There are $N$ equally sized electoral districts, indexed by $j$, where $N$ is odd, larger than 1. In a given district $j$ there is a share $\pi_{D j}$ of Democrats, a share $\pi_{R j}$ of Republicans, and a share $\pi_{I j}$ of Independents, with $\pi_{D j}+\pi_{R j}+\pi_{I j}=1$. The social planner decides the districting $\zeta$, i.e. how voters are distributed across the $N$ districts.

\footnotetext{
${ }^{3}$ This condition is met by all but two of the 48 contiguous States in the USA, according to the NYT-CBS annual polls and in all the States in our empirical analysis, but Rhode Island, which has an absolute majority (53\%) of Independents. These data are available at web site http://php.indiana.edu/ wright1/cbs7603_pct.zip.

${ }^{4}$ For the sake of our results, the number of seats in the legislature is irrelevant. We decided to assume a fixed number of districts as this mirrors more closely most of legislative assemblies, whose number of seats generally varies very rarely.
} 
Following Persson and Tabellini (2002) and Coate (2004), Independents' bliss points $\omega$ are uniformly distributed as $U[m-\tau, m+\tau] . m$ is a random variable distributed as $m \sim U[1 / 2-\epsilon, 1 / 2+\epsilon]$, which represent the ideology of the median Independent voter. The two distributions can be thought of as an idiosyncratic and an aggregate shock hitting the Independents' preferences, so that Independents are assumed to be on average (and ex-ante) centrist. The two distributions are common knowledge, but the realization of $m$ remains unknown to players.

We restrict the values that the uncertainty parameter $\epsilon$ can take, in order to avoid the possibility of an Independent being more extremist than party members, and to insure for any realization of $\tilde{m}$ there always is a share of left-leaning and a share of right-leaning Independents, moreover we assume that the "spread" of Independents is wide enough, coherently with anecdotal knowledge and with the empirical analysis. More specifically, the following assumptions are made:

Assumption 1. $\epsilon+\tau \leq 1 / 2 . \epsilon<1 / 4<\tau$.

Parties share the bliss point with their members, and in addition receive a positive rent $\Omega$ in case of victory. Parties are therefore simultaneously office- and policy-motivated. The Democratic (Republican) Party strategically chooses its policy platform $d(r)$ vis-à-vis the districting in order to maximize its expected utility. The Democratic and Republican Parties' expected utilities are defined as follows:

$$
\begin{aligned}
& E\left[U^{D}\right]=p[\Omega+L(0, d)]+(1-p) L(0, r) \\
& E\left[U^{R}\right]=p L(1, d)+(1-p)[\Omega+L(1, r)]
\end{aligned}
$$

where $p$ is the Democratic Party's probability of winning. As the expected utilities are continuous and defined over a compact set, existence of a Nash equilibrium is not an issue. ${ }^{5}$ In each district, parties field candidates that run on their Nash-equilibrium policy platforms $\left\{d^{*}, r^{*}\right\}$, and commit to implementing their policy platform in case of victory. The issue of the credibility of commitment to the publicly announced policy platform is beyond the scope of this paper; nevertheless we would assume that parties, following the standard citizen-candidate models (Osborne and Slivinski [1996], Besley and Coate [1997]), would commit to a particular policy through fielding a candidate whose preferences corresponded to the party's policy choice. Alternatively, we can think of the proposed platforms as the result of a repeated game with voters as in Alesina (1988).

In each district voters vote sincerely for the candidate with the policy that is nearer to their ideal point. The tie-breaking rule is a random draw. The candidate with the most votes is elected as a Representative. The Representatives then meet in the legislature and decide through majority voting the policy to implement between $d$ and $r$.

\footnotetext{
${ }^{5}$ The existence of multiple equilibria is also excluded. As we will see in the next section, in the linear case uniqueness is implied by the fact that the equilibrium sees parties playing strictly dominant strategies. The concave-utility case is instead solved numerically, and neither in our simulations nor through graphical analysis did we find evidence of the existence of any other equilibrium than the one described in Section 4.2.
} 
We can imagine that the districting, i.e. the way voters are distributed across districts, affects parties' platform choices and probabilities of winning. The districting $\zeta^{*}$ that maximizes the social welfare, defined as the sum of the utility of all voters:

$$
\begin{array}{r}
W(\zeta)=\sum_{i} E\left[u_{i}\left(d^{*}(\zeta), r^{*}(\zeta)\right)\right] \\
\zeta^{*}=\arg \max _{\zeta} W(\zeta) \text { s.t. } \zeta \in Z
\end{array}
$$

with $Z$ being the set of feasible districting. Intuitively, abstracting from geographical constraints, a districting is "feasible" if each voter belongs to one and only one district, and all districts are equally sized.

Finally, we define the correspondence between the optimal districting $\zeta^{*}$ and the seatvote curve $S\left(V \mid \zeta^{*}\right)$ generated by it. Summing up, the timing of the game is the following:

1. A districting plan $\zeta$ is implemented.

2. Parties commit to policies $\{d, r\} \in[0,1]$.

3. The position of the median voter $m$ is drawn from its distribution.

4. Citizens vote and in each district and the candidates with the most votes are elected.

5. The elected legislature votes to decide between the two policies and the one that obtains a majority is implemented.

The equilibrium concept is the subgame-perfect Nash equilbrium.

\section{Theoretical Results}

\section{Voters' and parties' behavior}

We focus our attention to cases in which the office-motivation parameter is not too large, limiting our attention on the interior solution in which policy platforms and probability of winning lie within the unit interval:

Assumption 2. For $\rho=1$, it is assumed that $\Omega<2 \epsilon$. For $\rho>1$, it is assumed $\Omega=0$.

Regarding the second part of the assumption, related to the cases of strictly convex preferences over policies, note that the results exposed in the remainder of this section would still hold in case the office-motivation parameter $\Omega$ were small enough. It will be shown how the model cannot be solved analytically but in the linear case $(\rho=1)$; this implies that it is not possible to explicitly state the threshold level for $\Omega$ which allows us to exclude the corner solutions from our analysis. Consequently, for the sake of clarity and simplicity we decided to focus on the case in which parties are solely policy-motivated as the convexity of preferences increases.

Let us focus our attention on a representative district $j$, which contains a share $\pi_{I j}$ of Independents, a share $\pi_{D j}$ of Democrats, and a share $\pi_{R j}$ of Republicans. In district $j$ there will be an Independent who is indifferent between voting Democrat or Republican, as her preferred policy is the midpoint between $d$ and $r$. The Democratic Party therefore carries the district if:

$$
\pi_{D j}+\pi_{I j} F\left(\frac{d+r}{2}, m\right)>\frac{1}{2}
$$


where $F(\cdot)$ is the cumulative distribution function of Independent voters, given the position of the median independent $m$. Knowing that $\pi_{R j}=\left(1-\pi_{D j}-\pi_{I j}\right)$, we can rearrange the expression bringing on the left-hand side the parameters related to the composition of the district:

$$
\frac{\pi_{D j}-\pi_{R j}}{\pi_{I j}}>1-2 \frac{\frac{r+d}{2}-m+\tau}{2 \tau}
$$

and define the variable $\kappa_{j}$ as the partisan slant or "Democratic leaning" of a district:

$$
\kappa_{j} \equiv \frac{\pi_{D j}-\pi_{R j}}{\pi_{I j}}
$$

Each district $j$ 's behavior is therefore described by the value of its partisan slant in favor of the Democrats $\kappa_{j}$. The Democratic Party carries this generic district if $\kappa_{j}$ is greater than the right hand side of (5).

This variable assumes value zero if the district has the same proportion of partisans of each ideology $\left(\pi_{D j}=\pi_{R j}\right)$ and its absolute value is inversely proportional to the amount of Independents. In addition through simple calculations we can see how a district with $\kappa_{j}>1$ is a safe Democratic district, i.e. a district in which the Democratic Party always obtains at least $50 \%$ of the votes. Similarly, a district with $\kappa_{j}<-1$ is a safe Republican district.

Ordering the districts according to their partisan slants $\kappa_{j}$, we can observe how the only district that matters in determining the implemented policy is the median one, that is the one determining the parliamentary majority. This means that given a median district's partisan slant $\tilde{\kappa}_{M}$, all the feasible districts $\zeta \in Z$ such that $\kappa_{M}=\tilde{\kappa}_{M}$ are equivalent from the point of view of parties and the social planner. They elicit the same strategic behavior from parties, and therefore give the same prescriptions to the social planner for the social optimum to be reached.

Starting from this consideration we can find a closed-form solution for the probability of winning of the Democratic Party.

Lemma 1. The probability $\mathrm{p}$ that the Democratic Party wins the election, conditional on parties' policy platforms $\{\mathrm{d}, \mathrm{r}\}$ is

$$
p(d, r)=\frac{1}{2}+\frac{d+r}{4 \epsilon}-\frac{1}{4 \epsilon}+\frac{\tau}{2 \epsilon} \kappa_{M}
$$

where $\kappa_{M}$ is the value of partisan slant of the median district in favor of the Democrats. Proof. See Appendix.

The Democratic Party's probability of winning depends on the districting per se $\left(\kappa_{M}\right)$ and on the parties' policy platforms. It increases the more the Democratic policy is to the right (in order to get more votes in the center), the more the Republican policy is 
extremist, and the more the median district is leaning towards the Democratic Party. ${ }^{6}$

Given this Lemma and parties expected utilities as in (2)-(3), we can find the equilibrium policy platforms. In the linear case, the equilibrium in dominant strategy can be found explicitly:

Proposition 1. As $\rho=1$, the Democratic and Republican Parties equilibrium policy platforms are respectively:

$$
\begin{aligned}
& d^{*}\left(\kappa_{M}\right)=\frac{1}{2}-\epsilon+\frac{1}{2} \Omega-\tau \kappa_{M} \\
& r^{*}\left(\kappa_{M}\right)=\frac{1}{2}+\epsilon-\frac{1}{2} \Omega-\tau \kappa_{M}
\end{aligned}
$$

\section{Proof. See Appendix.}

The equilibrium probability of winning therefore becomes $p^{*}=p\left(d^{*}, r^{*}, \rho=1\right)=1 / 2$.

As in Besley and Preston (2007), the districting, represented by the leaning of the median district $\kappa_{M}$, drags the proposed policies towards the same side of the districting ideological leaning. The office-motivation variable $\Omega$ drives the policies towards the center, in search of the vote of Independents. It must be noted how in this simpler linear case, the social planner decides the optimal districting $\kappa_{M}$ taking necessarily as a given the amount of policy divergence $\left(r^{*}-d^{*}\right)=(2 \epsilon-\Omega)$, which is unaffected by the social planner's decisions.

For the cases in which preferences are strictly convex $(\rho>1)$, it is not possible to find an analytical solution. Intuitively, each party best response is going to be affected by the fact that the marginal utility (loss) of moving one's policy platform towards the center increases more than proportionally with the distance between the platform and one's ideal point. Both of the platforms are affected by the districting, but the party that is disadvantaged by the districting (e.g. the Republicans if $\kappa_{M}>0$ ) needs to move its policy more decidedly away from its ideal point in order to try to recuperate its disadvantage. This in turn results in the party which is favored by the districting (e.g. the Democrats if $\kappa_{M}>0$ ) retaining its electoral advantage, and enjoying a probability of winning higher than 50\%. Moreover, in this general case, as the districting affects policy platform "asymmetrically", the social planner choice of $\kappa_{M}$ is going to affect policy divergence.

\section{Social Planner's behavior}

From the proof of Lemma 1 (in the Appendix), we can see that the median district (and with it the general election) is won by the Democratic Party as long as the position of the median voter $\tilde{m}$ falls to the left of a threshold level $\bar{m}$. More precisely the Democratic Party wins when: ${ }^{7}$

$$
\tilde{m}<\frac{d^{*}+r^{*}}{2}+\tau \kappa_{M} \equiv \bar{m}\left(\kappa_{M}\right)
$$

\footnotetext{
${ }^{6}$ In our analysis we shall restrict our attention to the value of $\kappa_{M}$ within the interval $[-1,1]$, i.e. the median district is not a "safe" district, the policy platforms and the probability of winning are interior solutions.

${ }^{7}$ In the linear-preference case $\bar{m}\left(\kappa_{M}\right)=1 / 2$.
} 
and the utility of a generic voter with ideology preference $i$ can be written as:

$$
\forall \tilde{m} \leq \bar{m}: u_{i}=L\left(i, d^{*}\right), \quad \forall \tilde{m}>\bar{m}: u_{i}=L\left(i, r^{*}\right)
$$

which implies that in expectations, her utility is:

$E\left[u_{i}\right]=\int_{1 / 2-\epsilon}^{\bar{m}} L\left(i, d^{*}\right) h(m) d m+\int_{\bar{m}}^{1 / 2+\epsilon} L\left(i, r^{*}\right) h(m) d m=p^{*} L\left(i, d^{*}\right)+\left(1-p^{*}\right) L\left(i, r^{*}\right)$

where $p^{*}$ is the equilibrium probability of winning of the Democratic party as from Lemma 1 , and $h(m)$ is the probability distribution function of the position of the median Independent $m$. Note also that $\{d, r, p, \bar{m}\}$ are affected by the districting $\kappa_{M}$. The social welfare function, i.e. the sum of all voters' expected utilities, can be written as:

$$
W\left(\kappa_{M}\right)=\bar{\pi}_{D} E\left[u_{0}\right]+\bar{\pi}_{R} E\left[u_{1}\right]+\bar{\pi}_{I} \int_{m-\tau}^{m+\tau} E\left[u_{i}\right] f(i) d i
$$

where $f(\cdot)$ is the probability distribution function of Independents $U[m-\tau, m+\tau]$.

The socially optimal districting, or the socially optimal median district (which is the same), is identified as the $\kappa_{M}$ that maximizes (10). The social planner takes as a given the equilibrium parties' best responses, the implied optimal (from the parties' utility point of view) divergence in policy platforms, and how this is affected by the districting $\kappa_{M}$. Given this, the aim of the social planner is to set up a districting such that (in expectation) voters' utility is maximized. This implies choosing a districting that in some way pushes parties to internalize the average ideological leaning of the population. In the simpler linear-preference case, this reduces to the social planner insuring that the average implemented policy is equal to the average preferred policy.

We define $\kappa_{M}^{*}$ as the socially optimal median district, and proceed in solving analytically the linear model.

Proposition 2. As $\rho=1$, in order to implement a welfare-maximizing districting, the median district must have a partisan slant $\kappa_{M}$ such that

$$
\kappa_{M}^{*}=\frac{\bar{\pi}_{D}-\bar{\pi}_{R}}{\bar{\pi}_{I}}
$$

as long as $\bar{\kappa} \equiv{ }^{\left(\bar{\pi}_{D}-\bar{\pi}_{R}\right)} / \bar{\pi}_{I} \in(a, b)$, with

$$
a=\max \left[-1+\frac{\Omega}{2 \tau},-1+\frac{2 \epsilon-\Omega}{2 \tau}\right], \quad b=\min \left[1-\frac{\Omega}{2 \tau}, 1-\frac{2 \epsilon-\Omega}{2 \tau}\right]
$$

When $\bar{\kappa}_{M} \notin[a, b]$, the socially optimal partisan slant $\kappa_{M}$ satisfies the following inequality:

$$
\begin{gathered}
\kappa_{M}^{*} \geq \frac{\bar{\pi}_{D}-\bar{\pi}_{R}}{\bar{\pi}_{I}} \text { if } \bar{\kappa} \in[b, 1] \\
\kappa_{M}^{*} \leq \frac{\bar{\pi}_{D}-\bar{\pi}_{R}}{\bar{\pi}_{I}} \text { if } \bar{\kappa} \in[-1, a]
\end{gathered}
$$


Proof. See Appendix.

The first part of the Proposition shows that in the linear case the welfare maximizing districting implies a (socially optimal) median district which is slanted in the same direction as the overall population leaning. Intuitively, given that parties shift their policy platforms to pander to the voters of the median district (see Proposition 1), the social planner must make the median district consonant with the whole nation's ideology, so to anticipate the parties' incentive to win the median-district. In the specific case in which preferences are linear, it translates into designing a median district that is a microcosm of the whole community, and reproduces exactly the population's ideological composition.

In case the distribution of ideologies in the population is particularly unbalanced (as in the second part of the Proposition), the socially optimal districting will imply a median district that is more "extremist" than the population as a whole. This depends primarily on technical reasons, which are relegated to the proof of Proposition 2.

For the more general non-linear case, as already mentioned, it is not possible to find an analytical solution. Nevertheless, we are able to find analytically how the median district should be designed in case preferences marginally deviate from the linear case. For the more comprehensive analysis of the equilibrium of the non-linear model we rely on numerical solutions.

Proposition 3. As the convexity of the preferences increases, in a neighborhood of the linear equilibrium, the optimal partisan slant of the median district decreases in absolute terms.

$$
\begin{array}{r}
\left.\frac{\mathrm{d} \kappa_{M}^{*}}{\mathrm{~d} \rho}\right|_{\left\{\kappa_{M}=\breve{\kappa}_{M}>0, \rho=1\right\}}<0,\left.\quad \frac{\mathrm{d} \kappa_{M}^{*}}{\mathrm{~d} \rho}\right|_{\left\{\kappa_{M}=\breve{\kappa}_{M}<0, \rho=1\right\}}>0, \\
\text { with } \breve{\kappa}_{M}=\frac{\left(\bar{\pi}_{D}-\bar{\pi}_{R}\right)}{\bar{\pi}_{I}}
\end{array}
$$

\section{Proof. See Appendix.}

This implies that as the convexity parameter $\rho$ increases, the socially optimal median district needs to be still slanted in favor of the most represented party in the society, but also needs to be "more centrist" than the population as a whole.

Numerical simulations allow us to generalize this result consistently with Proposition $3:$

Conjecture 1. For $\rho>1$, when voters have strictly convex preferences, there exist values of the parameters such that

$$
\begin{gathered}
0<\kappa_{M}^{*}<\frac{\bar{\pi}_{D}-\bar{\pi}_{R}}{\bar{\pi}_{I}} \text { if } \bar{\pi}_{D}>\bar{\pi}_{R} \\
\kappa_{M}^{*}>\frac{\bar{\pi}_{D}-\bar{\pi}_{R}}{\bar{\pi}_{I}}>0 \text { if } \bar{\pi}_{D}<\bar{\pi}_{R}
\end{gathered}
$$

We can therefore state with a good degree of certainty that in the case of strictly convex preferences the partisan slant of the socially optimal median district has to be consonant, 
but less extremist (or more centrist) than the population's ideological leaning (and therefore, than in the linear-model case). ${ }^{8}$ A sample of the simulation results is reported in Figure 1.

The electoral behavior that follows from Proposition 3 and Conjecture 1 could be summarized as follows. As in the linear case, parties' policy platforms are affected by the districting, and dragged in the same direction in which the population leans.

Unlike in the linear case, parties' platform will not be dragged to the same extent in the direction of the districting. As the utility function becomes more concave, parties' behavior becomes less responsive to the districting. These two effects imply that the indifferent voter is more centrist than in the linear case, and the vote share accruing to each party reflects more closely the population composition: the party with a larger partisan base receives an absolute majority of votes.

Finally, some words are still needed on the feasibility of the socially optimal districting. One may notice that there are two distinct aspects of feasibility: firstly, the districting must be such that each voter belongs to one and only one district, and that each district contains the same number of voters. In this sense the constraint that the social planner faces is the ideological composition of the population. The second - and more stringenttype of feasibility arises from the geographical distribution of Democratic, Republican and Independent voters in the State. Under this definition, a districting is feasible if the geographical distribution of voters and the legal constraints on shape and connectedness allow the social planner to design a median district as prescribed by the optimality conditions.

With regards to the first definition given, the optimality condition on the median district imposed by Proposition 2 is always feasible. The most intuitive example for this would be to have the so-called "uniform districting," i.e. having all the districts with the exact same composition, which is of course the general population's composition as well. For what concerns the feasibility in case preferences are not linear, a more formal discussion is given in the Appendix.

This paper, as most of the literature on districting, instead does not focus on the issues arising from the geographical distribution of voters within a state, and therefore abstracts from possible problems of geographical feasibility. Notable exceptions are Sherstyuk (1998) and Puppe and Tasndi (2009). Anecdotally, one can observe the large number of "oddly-shaped" districts, which appear to seek to include (or exclude) specific neighborhoods, or run lengthly un-inhabited territories to join distant parts of the same state within the one district. ${ }^{9}$ This seems to point towards the fact that the increased

\footnotetext{
${ }^{8}$ The unicity of the pure-strategy Nash equilibrium has also been confirmed. The simulations are performed focusing on the cases where $\rho \in[1,10]$ and $\tau \geq 0.3$. This last restriction allows us to focus on cases in which the Independents' preferences are sufficiently heterogeneous, which is consistent with anecdotal knowledge and with the findings of our empirical analysis in Section 4. These results also hold as long as the office-motivation parameter $\Omega$ is small enough. Higher values of the parameter $\Omega$ may have two distinct effects. First, it may lead us to corner solutions in which Democratic and Republican policy platforms converge. Secondly, in some cases in which the population's ideological distribution is very unbalanced and the concavity parameter $\rho$ is very high the social planner may need to reward the largest party in order to reach the social optimum, giving us opposite results from the one exposed in Conjecture 1.

${ }^{9}$ For a good report on the issue, see: "How to Rig an Election", The Economist, April 25th 2002.
} 
data availability, and computational possibilities are allowing districting authorities to overcome geographical and informational constraints drawing the boundaries with - so to say-finer and finer lines.

\section{The Seat-Vote Curve}

The seat-vote curve is a mathematical relation $S(V)$ that links the number of votes $V$ obtained by a party at the national level with the amount of seats $S$ won in the legislature. As the policy to be implemented is decided by majority voting in the legislature, i.e. by the median legislator, from the welfare point of view it is irrelevant whether a party has a slim or an overwhelming majority of seats: the implemented policy would be the same one. For this reason it does not exist a unique seat-vote curve that would maximize social welfare.

It is possible, however, to investigate which party carries the median district (i.e. obtains a majority in the legislature) when both parties obtain half of the votes at the national level. In this way we can pin down the position of the seat-vote curve in one point $(V=1 / 2)$, and determine the direction of the bias.

The bias of the seat-vote curve is defined as $\alpha=S(1 / 2)-1 / 2$. The value of the bias $\alpha$ represents how many seats over the cut-off of $50 \%$ the Democratic Party obtains in correspondence to $50 \%$ of the votes.

When each party obtains $50 \%$ of votes at the national level, the party which wins in the median district enjoys a bias in its favor, having obtained an absolute majority of seats with only half of the votes.

We define a bias as "reinforcing" if it favors the party with the largest partisan base, and "counterbalancing" if it favors the smallest partisan group, i.e.

$$
\begin{array}{r}
\text { reinforcing: if }\left\{\begin{array}{l}
\alpha>0 \text { and } \bar{\pi}_{D}>\bar{\pi}_{R} \\
\alpha<0 \text { and } \bar{\pi}_{D}<\bar{\pi}_{R}
\end{array}\right. \\
\text { counterbalancing: if }\left\{\begin{array}{l}
\alpha>0 \text { and } \bar{\pi}_{D}<\bar{\pi}_{R} \\
\alpha<0 \text { and } \bar{\pi}_{D}>\bar{\pi}_{R}
\end{array}\right.
\end{array}
$$

This leads us to the most important findings of this paper:

Proposition 4. In the case of linear preferences $(\rho=1)$, the seat-vote curve is unbiased $(\alpha=0)$ as the socially optimal districting is implemented and the population's ideology is not too unbalanced $(\bar{\kappa} \in[a, b])$.

If the population has a very skewed ideological distribution ( $\bar{\kappa} \notin[a, b]$ ), the optimal seat-vote curve will be biased in favor of the party most represented in the population (reinforcing bias).

Proof. See Appendix.

In the linear preference case, if the population is ideologically "balanced enough", the socially optimal seat-vote curve must not be biased: no party should get any advantage from the districting. When the population composition is very extremist, the bias will be reinforcing, i.e. it will favor the party that has the larger popular support. 
From Proposition 2 we know that as long as the population is not too extremist, in equilibrium the median district will be a microcosm of the whole population, therefore its voting behavior will exactly reflect the national voting pattern. If the parties, at the national level, each obtain half of the vote, the same will happen in the median district, with each party expecting to obtain a majority in the legislature with $50 \%$ probability. This implies an unbiased seat-vote curve. In other words, as policy platforms already match the population ideological composition, the social planner has no reason to skew the seat-vote curve in any direction.

This is in line with what would have happened to the results of Coate and Knight (2007), had they considered the linear case. As we further explore the effect on the seatvote curve of having players with strictly convex preferences, we are able to state the second major finding of the paper:

Lemma 2. If $\bar{\kappa} \in[a, b]$, in a neighborhood of the linear equilibrium, the socially optimal seat-vote curve shows a counterbalancing bias when preferences are strictly convex.

Proof. See Appendix.

Conjecture 2. When voters and parties have strictly convex preferences (i.e. for $\rho>2$ ), there exist values of the parameters such that the socially optimal seat-vote curve's bias is counterbalancing.

This result is exactly the opposite of Coate and Knight's (2007). In their paper the social planner has to support the party with a larger partisan base. This is so that the implemented policy, which moves quite rigidly as the legislature composition changes, corresponds to the social optimum.

In our setup, as parties endogenously adapt their policies to the districting, the social planner will construct a districting that is consonant with the same ideological leaning, albeit more centrist than the population as a whole (see Proposition 3 and Conjecture 1), and parties' policy platforms will move accordingly. As a consequence, the minority party will (mostly) recuperate its ex-ante electoral disadvantage in term of seats, but the majoritarian party will still retain a large majority of the national vote share. The effect on the seat-vote curve is to have a counterbalancing bias, in which a large majority of votes at the national level translates in a small parliamentary majority in terms of seats.

This happens because of the interplay of parties endogenously choosing their platforms with the concavity of voters' utility function, which makes the social optimum closer to the ideological center. Allowing parties to endogenously condition their policy platforms on the districting implies that the social planner must counterbalance the tendency of parties to move their platforms too decidedly away from the center, as they follow the districting. As it is briefly analyzed in a theoretical extension included in the Appendix, this feature holds even if the implemented policy were to be formed through parliamentary bargaining rather than majority voting.

\section{Empirical Analysis}

In this section we estimate the possible welfare gain from implementing the socially optimal districting when preferences are convex, so to compare our results with the ones 
previously obtained in the literature. ${ }^{10}$

To make a closer comparison with Coate and Knight (2007), we will refer to the specific case in which preferences are quadratic $(\rho=2)$, and we use their same dataset, which has been kindly provided by Brian Knight. The data include the electoral results of the elections of State Lower Houses, for the 5 electoral rounds held after the redistricting that followed the 1990 Census $(1992,1994,1996,1998$ and 2000), together with demographic data on each district's population (e.g. average household income, percentage of African-Americans, elderly people, college graduates, share of urban, suburban and rural population). We also use State-level polling data from the already-cited New York Times-CBS survey (see footnote 4), in which a representative sample of voters is annually asked to self-identify as Democrat, Republican or Independent. For the purpose of this paper the 1991-2000 average level of self-identification as Democrat, Republican, or Independent in each of the 28 States is used.

The welfare under the optimal districting $W^{\text {opt }}$ and under the actual districting $W^{\text {act }}$ are calculated using the formula as in (10). As the value of the social welfare is within the $[-1,0]$ interval, we define the welfare gain as: ${ }^{11}$

$$
W^{\text {gain }}=\frac{\left(1+W^{\text {opt }}\right)-\left(1+W^{\text {act }}\right)}{1+W^{\text {act }}}
$$

From the raw data just described, we are in possession only of the shares of Democrats $\left(\bar{\pi}_{D}\right)$, Republicans $\left(\bar{\pi}_{R}\right)$ and Independents $\left(\bar{\pi}_{I}\right)$ for each State, and the electoral results for each district in each election. We instead need to estimate the true moments (mean and variance) of the Democratic vote share in each district, and the uncertainty parameters $(\epsilon, \tau)$, and rely on our theoretical model in order to calculate the welfare levels under the actual and optimal districting.

\subsection{Empirical Approach}

The share of votes $V_{j}$ earned by the Democratic Party in a representative district $j$ is

$$
V_{j}=\pi_{D j}+\frac{\pi_{I j}}{2 \tau}(x-m+\tau), \quad \text { with } \quad x=\frac{d+r}{2}
$$

\footnotetext{
${ }^{10}$ The same procedure applied to the linear-preference model gives us very similar results, showing very small welfare gains from implementing the socially optimal districting.

${ }^{11}$ Coate and Knight (2007) defined voters' utility as$$
U_{i}=\gamma+\beta L(i, x)
$$

assuming $\beta / \gamma \geq 1$; we instead assumed a simpler functional form: $U_{i}=L(i, x)$. According to their (more general) utility's functional form the value of welfare gain we find through (14) would be the maximum welfare gain. Choosing to use our simpler functional form, implicitly implies assuming that $\beta / \gamma=1$. Our results about the welfare gain are perfectly comparable with the one by Coate and Knight (2007). In fact in their paper they mostly refer to the maximimum welfare gains, which correspond to the case when $\beta / \gamma=1$.
} 
Only data on $V_{j}$ are included in the dataset; all other parameters of (15) need to be estimated. Knowing the distribution of $m$, we can compute the first two moments of the Democratic vote share distribution at the district- $j$ 's $\left(V_{j}\right)$ and national $(V)$ level:

$$
\begin{array}{cc}
E\left[V_{j}\right]=\pi_{D j}+\frac{1}{2 \tau} \pi_{I j}+\frac{\pi_{I j}}{2 \tau}\left(x-\frac{1}{2}\right), & \sigma_{j}=\frac{\pi_{I j} \epsilon}{2 \sqrt{3} \tau} \\
E[V]=\pi_{D}+\frac{1}{2 \tau} \pi_{I}+\frac{\pi_{I}}{2 \tau}\left(x-\frac{1}{2}\right), & \sigma_{V}=\frac{\bar{\pi}_{I} \epsilon}{2 \sqrt{3} \tau}
\end{array}
$$

With a sufficiently long panel, the estimation of moments would simply consist of reporting the sample average and standard error. In the U.S. though, redistricting happens every ten years, and therefore for each district our database contains at most five observations. Moreover there is a non-negligible number of uncontested districts, i.e. districts in which in all the five election rounds only one candidate ran. We therefore track the methodology used in Coate and Knight (2007), calculating the districts' moments as a function of the demographic characteristics of each district, using a bootstrapping technique over the following random-effect model with heteroscedasticity:

$$
V_{j t}=X_{j}^{\prime} \beta_{1}+\sigma_{1} \xi_{j}+u_{j t}, \quad \log \left(u_{j t}^{2}\right)=X_{j}^{\prime} \beta_{2}+\sigma_{2} \nu_{j}+\log \left(\omega_{t}^{2}\right)
$$

where $V_{j t}$ is the share of votes for the Democratic Party in district $j$ at the election round $t$, $X_{j}$ is the matrix of controls, including the demographic data on the district and the Statelevel polls, and State dummies, $\beta_{1}$ and $\beta_{2}$ are the parameters to be estimated, and $\xi_{j}$ and $\nu_{j}$ are the district-specific random effects, which are assumed to be normally distributed and with standard error $\sigma_{\xi}$ and $\sigma_{\nu}$, respectively. The last terms in the two equation are the usual white noise. The estimation technique is a standard two-step approach

To know the district-specific moments, two sources of uncertainty are to be controlled: first of all we have the estimated and not the true values of the parameters of the two regressions $\left(\beta_{1}, \beta_{2}, \sigma_{1}, \sigma_{2}\right)$; secondly, we do not observe the random effects $\left(\xi_{j}, \nu_{j}\right)$. That is why a bootstrapping technique is used: for each replication $r=1,2, \ldots, 100$ a sample of size $N$ is drawn with replacement from the dataset of $N$ districts, where $N$ is the number of districts with at least one contested election. Then the estimation with the standard two-step approach is performed for each of the 100 samples. In this way we obtain the entire distribution of the parameter estimates $\left(\beta_{1}^{r}, \beta_{2}^{r}, \sigma_{1}^{r}, \sigma_{2}^{r}\right)_{r=1}^{r=100}$. To learn the district-specific mean and standard error we need a further step in order to take the random effects into account. We therefore draw from a standard normal distribution the random effects $\left(\xi_{j}^{r}, \nu_{j}^{r}\right)$ for each replication $r$ and each district $j$. Thus we can calculate the district-specific mean and variance:

$$
E\left[V_{j}\right]^{r}=X_{j}^{\prime} \beta_{1}^{r}+\sigma_{1}^{r} \xi_{j}^{r}, \quad \sigma_{j}^{r}=\sqrt{\exp \left(X_{j}^{\prime} \beta_{2}^{r}+\sigma_{2}^{r} \nu_{j}^{r}\right)}
$$

We are then able to calculate the State-specific moments averaging across districts, and use the values predicted by the central moment of the simulated parameters to obtain the 
results for the uncontested districts.

\subsection{Calibration of the Theoretical Model}

In line with Coate and Knight (2007), ${ }^{12}$ we assume that $\epsilon+\tau=1 / 2$. Let's recall (17), which describes the standard deviation of the votes for the Democratic Party in a particular State.

$$
\widehat{\sigma}_{V}=\frac{\bar{\pi}_{I} \epsilon}{2 \sqrt{3} \tau}
$$

$\sigma_{V}$ is obtained from the bootstrap procedure just described, $\bar{\pi}_{I}$ is instead assumed to be equal to the data from the NYT-CBS polls. From (20) we can obtain the State-level results for $\widetilde{\epsilon}$ and $\widetilde{\tau}$, and calculate the district-specific $\widetilde{\pi}_{I j}$ :

$$
\tilde{\pi}_{I j}=\frac{2 \sqrt{3} \widetilde{\tau} \widehat{\sigma}_{j}^{r}}{\widetilde{\epsilon}}
$$

From the expression for $E[V]$ :

$$
\widehat{E[V]}=\bar{\pi}_{D}+\frac{1}{2 \tilde{\tau}} \bar{\pi}_{I}+\frac{\bar{\pi}_{I}}{2 \tilde{\tau}}\left(x-\frac{1}{2}\right)
$$

we can calculate the policies midpoint $\tilde{x}$. From this we are able to calculate Democratic slant and parties' policy platforms implied by the voting distribution in each state:

$$
=\left\{d, r, \kappa \mid \frac{\partial E\left[U^{D}\right]}{\partial d}=0, \frac{\partial E\left[U^{R}\right]}{\partial r}=0, \frac{\partial^{2} E\left[U^{D}\right]}{\partial d^{2}}<0, \frac{\partial^{2} E\left[U^{R}\right]}{\partial r^{2}}<0, \frac{d+r}{2}=\tilde{x}\right\}
$$

With $\left\{\tilde{d}^{a c t}, \tilde{r}^{a c t}, \tilde{\kappa}_{M}^{a c t}\right\}$ in hands, we are able to calculate the welfare under the actual districting as from equation (10). Moreover, we are also able to calculate the optimal Democratic slant of the median district $\kappa_{M}^{*}$, and the welfare corresponding to the optimal districting. All of these calculations are performed for each of the 28 States in our dataset and for each one of the one hundred replications in our simulations.

Our empirical analysis does not unveil any evident regularity regarding the districting bias (or Democratic slant). The difference between the actual and optimal median district composition in each State as observed in Table 1 does not seem to follow a pattern. This in a way confirms the inconcluding results of Coate and Knight (2007) and more generally the very variable results found in the literature regarding the presence and direction of a bias in the electoral system.

The welfare gains, as showed in Table 2, appear consistently small across States. For twenty out of 28 States the welfare gain is solidly below $1 \%$ and only for three States

\footnotetext{
${ }^{12} \mathrm{As}$ in Coate and Knight (2007), the same analysis is performed also assuming $\epsilon+\tau=\bar{z}<.5$, and gives absolutely analogous results to the ones reported in this section, which are available upon request.
} 
the welfare gain is larger than $3 \%$. The magnitude of the welfare gain is even smaller than the one found by Coate and Knight (2007), where parties were constrained to run on extremist platforms. Our empirical finding therefore strongly reinforces Coate and Knight (2007), when they state that a shift towards a districting properly laid down by a benevolent social planner would not significantly increase the overall population's welfare.

This may be due to the fact that - irrespective of the districting - parties already choose to run on policies which are quite "centrist", in search of the Independents' vote. If this were the case, passing from the actual to the optimal districting may end up having a relatively small effect on policies, and therefore on welfare. This finding of course relies heavily on our modeling choices, and especially on the assumption that each candidate of a given party runs on the same policy platform irrespective of its district composition.

For what concerns the few outliers that show a stronger welfare gain, this may be imputed to some form of measurement error in our data. For example the NYT-CBS poll of self-identification as Democrat, Republican or Independent may not reflect the population preferences homogeneously across States. In other words, self-declared Independent voters in some States may not be "on average centrist" as we assumed in our model. This may be particularly true in States with very skewed ideological distributions, or a particular political history.

\section{Concluding remarks}

This paper analyzed socially optimal districting in a framework in which parties endogenously choose nationwide policy platforms vis-à-vis districting. Parties react strategically to districting, presenting policy platforms that will be more liberal the more the districting leans towards the Democrats. We investigated what the districting should be in order to reach the welfare optimum. Secondly, we analyzed which seat-vote curve is consistent with the social optimum.

Our analysis shows that the social planner should design the districting so that the median district's composition reflects the population's ideological leaning, in order to bring the expected implemented policy towards the welfare maximizing policy.

The consequent seat-vote curve that stems from this finding is unbiased in the linearpreference case. In the more general case in which voters and parties have concave utilities, the optimal seat-vote curve instead needs to be biased against the largest partisan group; the social planner ought to draw a median district which is more centrist than the population, albeit still leaning in the same direction. Consequently the policy proposals will - even if less decidedly than in the linear case - lean towards the same side as the population (and the districting). The party with a larger partisan base will get more than half of the votes and will expect to win just over $50 \%$ of the seats. For this reason the seat-vote curve will show a counterbalancing bias, i.e. a bias against the larger party in the population. The results regarding the bias of the seat-vote curve hold also in those cases where the party caucuses in the legislature bargain over the policy to implement; the bias remains null if preferences are linear and counterbalancing if voters have concave utilities.

These results strongly contradict Coate and Knight's (2007) prescriptions regarding the socially optimal districting. In their seminal paper they modeled a polity in which 
parties' platform are exogenous, but the implemented policy is the result of a "bargaining" process in which the chosen policy is the average of the exogenous party platforms weighted by their seat share, i.e., the policy which maximizes the legislators' joint utility. In such a setup, the optimal seat-vote curve is unbiased when preferences are linear, but requires a reinforcing bias when utilities are concave.

An empirical calibration of the base model is performed on data from the U.S. State Lower Houses' elections of 28 States for 5 electoral rounds (1992 to 2000). It appears that implementing the socially optimal districting would indeed increase the social welfare, but the size of this effect is even smaller than the one found by Coate and Knight (2007). Letting parties condition their policy platforms on the districting naturally constrains them to take Independents' preferences into greater account. This in turn makes the size of the potential welfare gain from implementing the optimal districting very small: in the majority of States it remains below $1 \%$.

Further research is needed in order to analyze in greater depth the political economy of districting and its policy and welfare consequences. The effects of migration across districts, the microfoundation of the process through which the legislature decides the implemented policy and the political-economy motivations that lead politicians to devolve or not the power of redistricting to independent commissions have yet to be analyzed.

\section{Acknowledgements}

I am grateful to Amrita Dhillon and Ben Lockwood for their continual advice. I thank as well Tim Besley, Steve Coate and Brian Knight, three anonymous referees and the participants of seminars held at the University of Warwick, the World Conference of the Game Theory Society at Northwestern University, the EEA Annual Meeting in Milan and the RES Annual Conference in Surrey for useful comments. The usual disclaimer applies. 


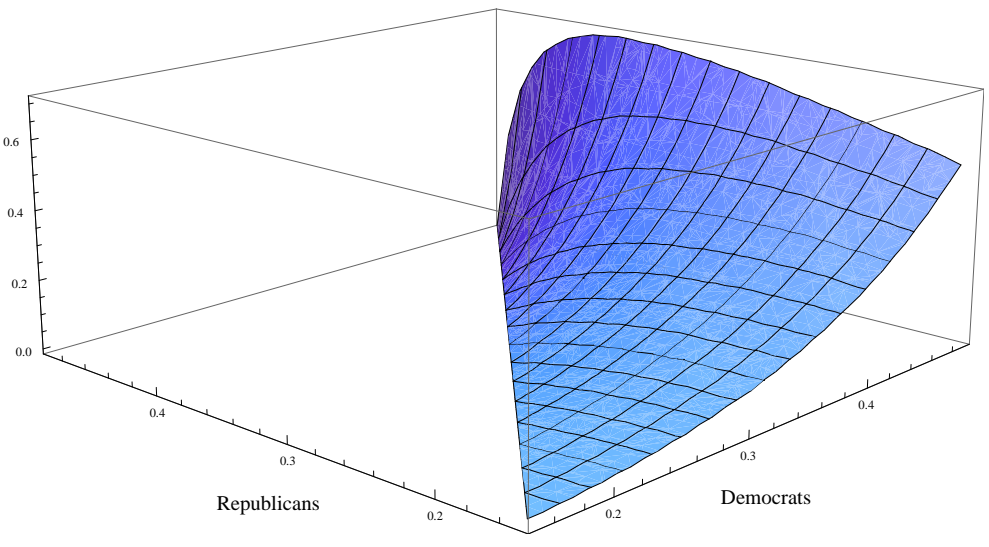

Figure 1: Difference between linear-preference (as in Proposition 2) and quadratic-preference (as from Proposition 3 and Conjecture 1, numerical solutions) optimal median-district Democratic slant. Calculated for $\epsilon=.1, \tau=.4$, and for $\bar{\pi}_{D} \geq \bar{\pi}_{R}$. Note how the difference is always positive, i.e. $\kappa_{M}^{*}(\rho=1)>\kappa_{M}^{*}(\rho=2)$. 


\begin{tabular}{|l|cc|c|c|ccc|}
\hline State & $\bar{\pi}_{D}$ & $\bar{\pi}_{R}$ & $\kappa_{M}^{\text {opt }}, \rho=1$ & $\kappa_{M}^{\text {opt }}, \rho=2$ & \multicolumn{3}{|c|}{$\kappa_{M}^{\text {act }}, \rho=2$} \\
& & & & & mean & median & $90 \%$ C.I. \\
\hline \hline AL & $36.77 \%$ & $31.44 \%$ & 0.168 & 0.060 & -0.285 & -0.282 & {$[-0.421,-0.179]$} \\
CA & $40.11 \%$ & $34.92 \%$ & 0.208 & 0.090 & -0.346 & -0.343 & {$[-0.61,-0.103]$} \\
CO & $28.84 \%$ & $32.88 \%$ & -0.106 & -0.050 & 0.101 & 0.097 & {$[0.027,0.193]$} \\
CT & $31.77 \%$ & $27.07 \%$ & 0.114 & 0.063 & -0.104 & -0.103 & {$[-0.16,-0.049]$} \\
DE & $37.41 \%$ & $28.82 \%$ & 0.254 & 0.119 & 0.407 & 0.408 & {$[0.269,0.581]$} \\
FL & $37.03 \%$ & $35.43 \%$ & 0.058 & 0.020 & 0.041 & 0.043 & {$[-0.033,0.112]$} \\
IA & $31.00 \%$ & $29.69 \%$ & 0.033 & 0.020 & 0.072 & 0.070 & {$[-0.002,0.143]$} \\
IL & $36.53 \%$ & $29.16 \%$ & 0.215 & 0.090 & -0.073 & -0.076 & {$[-0.127,-0.02]$} \\
KS & $28.18 \%$ & $40.56 \%$ & -0.396 & -0.172 & -0.112 & -0.113 & {$[-0.191,-0.023]$} \\
KY & $46.69 \%$ & $31.43 \%$ & 0.697 & 0.199 & 0.237 & 0.236 & {$[0.126,0.364]$} \\
ME & $25.66 \%$ & $29.50 \%$ & -0.086 & -0.050 & -0.199 & -0.197 & {$[-0.266,-0.142]$} \\
MI & $33.86 \%$ & $30.35 \%$ & 0.098 & 0.040 & -0.085 & -0.089 & {$[-0.125,-0.035]$} \\
MO & $35.20 \%$ & $28.32 \%$ & 0.189 & 0.090 & -0.010 & -0.008 & {$[-0.072,0.053]$} \\
MS & $39.78 \%$ & $35.25 \%$ & 0.181 & 0.060 & -0.527 & -0.519 & {$[-0.701,-0.359]$} \\
MT & $29.62 \%$ & $33.97 \%$ & -0.119 & -0.058 & 0.153 & 0.151 & {$[0.062,0.242]$} \\
NM & $37.08 \%$ & $35.58 \%$ & 0.055 & 0.020 & -0.305 & -0.307 & {$[-0.454,-0.16]$} \\
NV & $32.29 \%$ & $39.61 \%$ & -0.260 & -0.100 & -0.459 & -0.464 & {$[-0.6,-0.298]$} \\
NY & $40.23 \%$ & $29.46 \%$ & 0.355 & 0.130 & -0.410 & -0.412 & {$[-0.463,-0.365]$} \\
OH & $35.62 \%$ & $32.17 \%$ & 0.107 & 0.040 & 0.093 & 0.090 & {$[0.049,0.145]$} \\
OK & $48.33 \%$ & $33.52 \%$ & 0.816 & 0.237 & 0.327 & 0.331 & {$[0.107,0.524]$} \\
OR & $36.56 \%$ & $33.82 \%$ & 0.093 & 0.034 & 0.095 & 0.096 & {$[-0.007,0.199]$} \\
PA & $40.12 \%$ & $36.08 \%$ & 0.170 & 0.059 & 0.071 & 0.067 & {$[-0.036,0.163]$} \\
RI & $30.76 \%$ & $17.13 \%$ & 0.262 & 0.161 & -0.244 & -0.239 & {$[-0.299,-0.19]$} \\
SC & $33.28 \%$ & $35.65 \%$ & -0.076 & -0.030 & -0.115 & -0.113 & {$[-0.213,-0.023]$} \\
TN & $35.62 \%$ & $31.64 \%$ & 0.122 & 0.050 & -0.057 & -0.048 & {$[-0.165,0.026]$} \\
UT & $21.59 \%$ & $48.12 \%$ & -0.876 & -0.351 & -0.320 & -0.320 & {$[-0.433,-0.207]$} \\
VA & $32.18 \%$ & $33.66 \%$ & -0.043 & -0.020 & -0.061 & -0.063 & {$[-0.135,0.022]$} \\
WI & $32.88 \%$ & $30.19 \%$ & 0.073 & 0.030 & 0.080 & 0.079 & {$[0.01,0.141]$} \\
\hline
\end{tabular}

Table 1: Calibrated Optimal and Actual Median District Democratic Slant by State

\begin{tabular}{|c|c|c|c|}
\hline State & Mean & Median & $\mathbf{9 0 \%}$ C.I. \\
\hline CT & $0.482 \%$ & $0.458 \%$ & {$[0.206 \%, 0.823 \%]$} \\
ME & $0.397 \%$ & $0.365 \%$ & {$[0.141 \%, 0.77 \%]$} \\
RI & $3.608 \%$ & $3.536 \%$ & {$[2.68 \%, 4.689 \%]$} \\
DE & $1.516 \%$ & $1.359 \%$ & {$[0.475 \%, 3.718 \%]$} \\
NY & $6.883 \%$ & $6.908 \%$ & {$[5.648 \%, 8.249 \%]$} \\
PA & $0.070 \%$ & $0.038 \%$ & {$[0 \%, 0.214 \%]$} \\
IL & $0.588 \%$ & $0.592 \%$ & {$[0.253 \%, 1.017 \%]$} \\
MI & $0.373 \%$ & $0.381 \%$ & {$[0.13 \%, 0.616 \%]$} \\
OH & $0.079 \%$ & $0.055 \%$ & {$[0.002 \%, 0.241 \%]$} \\
WI & $0.083 \%$ & $0.050 \%$ & {$[0.001 \%, 0.248 \%]$} \\
IA & $0.100 \%$ & $0.059 \%$ & {$[0.001 \%, 0.314 \%]$} \\
KS & $0.103 \%$ & $0.061 \%$ & {$[0 \%, 0.364 \%]$} \\
MO & $0.211 \%$ & $0.173 \%$ & {$[0.023 \%, 0.493 \%]$} \\
VA & $0.081 \%$ & $0.052 \%$ & {$[0 \%, 0.273 \%]$} \\
AL & $2.910 \%$ & $2.736 \%$ & {$[1.316 \%, 5.577 \%]$} \\
FL & $0.062 \%$ & $0.038 \%$ & {$[0.001 \%, 0.218 \%]$} \\
MS & $7.403 \%$ & $7.037 \%$ & {$[3.387 \%, 12.768 \%]$} \\
SC & $0.220 \%$ & $0.144 \%$ & {$[0.004 \%, 0.717 \%]$} \\
KY & $0.123 \%$ & $0.080 \%$ & {$[0.001 \%, 0.499 \%]$} \\
OK & $0.285 \%$ & $0.166 \%$ & {$[0.002 \%, 1.127 \%]$} \\
TN & $0.318 \%$ & $0.190 \%$ & {$[0.022 \%, 0.964 \%]$} \\
CO & $0.545 \%$ & $0.473 \%$ & {$[0.125 \%, 1.266 \%]$} \\
MT & $0.884 \%$ & $0.831 \%$ & {$[0.263 \%, 1.69 \%]$} \\
NV & $2.356 \%$ & $2.250 \%$ & {$[0.695 \%, 4.348 \%]$} \\
NM & $2.143 \%$ & $2.008 \%$ & {$[0.593 \%, 4.233 \%]$} \\
UT & $0.092 \%$ & $0.048 \%$ & {$[0 \%, 0.387 \%]$} \\
CA & $2.411 \%$ & $2.075 \%$ & {$[0.401 \%, 5.517 \%]$} \\
OR & $0.150 \%$ & $0.085 \%$ & {$[0.001 \%, 0.531 \%]$} \\
\hline
\end{tabular}

Table 2: Welfare Gain by State (mean, median and $90 \%$ confidence interval) 


\section{APPENDIX}

\section{Coate and Knight (2007) with risk-neutral voters}

From Coate and Knight (2007) we know that the share of votes obtained by the Democratic Party is

$$
V=\bar{\pi}_{D}+\frac{\bar{\pi}_{I}}{2 \tau}\left(\frac{1}{2}-m+\tau\right) \text {, i.e. } m=\frac{1}{2}+\tau\left(\frac{\bar{\pi}_{I}+2 \bar{\pi}_{D}-2 V}{\bar{\pi}_{I}}\right)
$$

The implemented policy is $1-S$, weighted average of 0 and 1 by the seat share $S$. The social welfare function $W$ is:

$$
W=-\left[\bar{\pi}_{D}(1-S)+\bar{\pi}_{R} S+\frac{\bar{\pi}_{I}}{2 \tau} \int_{m-\tau}^{m+\tau}|(1-S)-x| d x\right]
$$

Maximising $W$ with respect to $S$, and taking (A.1) into account:

$$
\underset{S}{\arg \max } W=\frac{1}{2}+\frac{2 \tau}{\bar{\pi}_{I}}\left(V-\frac{1}{2}\right)
$$

which is an unbiased seat-vote curve.

\section{Extension to the Theoretical Model: Legislative Bargaining à la Coate and Knight (2007)}

In order to have a closer comparison with Coate and Knight (2007), we check if our results exposed in Section 4 hold in case the legislature decided through the bargaining process as described in Coate and Knight (2007), in case $\rho \in\{1,2\}$ and $\Omega=0$. In their model, parties represent the two extreme policy platforms ( 0 and 1$)$, and the legislature implements a policy which is the average of the two parties' platforms weighted by their seat shares.

As in Coate and Knight (2007), we assume that voters vote "naively" for the party proposing the policy nearer to their ideal point, not taking into considerations forms of strategic voting in which citizens anticipate the bargaining in the legislature.

The timing of the game could be therefore be described as follows:

1. The districting is implemented.

2. Parties propose their policies $\{d, r\}$.

3. Voters vote in each district for the party whose policy is closer to their bliss point.

4. The legislature is elected.

5. Bargaining: the implemented policy $x=S d+(1-S) r$ is the average between the proposed ones, weighted by the seats of each party, where $S$ is the seat share won by Democrats.

We define parties' preferences as:

$$
U^{D}=-x^{\rho}, U^{R}=-(1-x)^{\rho}, \text { with } x=S d+(1-S) r
$$


We also assume a linear functional form: ${ }^{13}$

$$
S=\frac{1}{2}+a+b\left(V-\frac{1}{2}\right), \text { with } V=\bar{\pi}_{D}+\frac{\bar{\pi}_{I}}{2}+\frac{\bar{\pi}_{I}}{2 \tau}\left(\frac{r+d}{2}-\frac{1}{2}\right)
$$

Plugging (A.5) into (A.4), we can maximize each party's utility function with respect to its policy proposal and obtain parties' equilibrium policy platforms.

Proposition 5. If the implemented policy is decided through parliamentary bargaining, and the seat-vote curve is as in (A.5), the interior solution for parties' equilibrium policies is:

$$
\begin{aligned}
& d^{*}=\frac{1}{2}-\frac{\tau}{\bar{\pi}_{I}}\left(\bar{\pi}_{D}-\bar{\pi}_{R}\right)-\frac{\tau(2 a+1)}{b \bar{\pi}_{I}} \\
& r^{*}=\frac{1}{2}-\frac{\tau}{\bar{\pi}_{I}}\left(\bar{\pi}_{D}-\bar{\pi}_{R}\right)-\frac{\tau(2 a-1)}{b \bar{\pi}_{I}}
\end{aligned}
$$

independently of voters' attitude towards risk.

Proof. Given (A.5), substituting $V$ into $S(V)$, then $S$ into (A.4), we obtain the fully explicit (and concave) utility functions of each party. The Nash-equilibrium (dominant strategy) platforms are derived solving the first order conditions of (A.4):

$$
\frac{\partial U^{D}}{\partial d}:-\rho x^{\rho-1} \cdot \frac{\partial x}{\partial d}=\frac{\partial x}{\partial d}=0, \quad \frac{\partial U^{D}}{\partial r}: \rho(1-x)^{\rho-1} \cdot \frac{\partial x}{\partial r}=\frac{\partial x}{\partial r}=0
$$

whose solution is independent of $\rho, \forall x \neq 0$

Corollary 1. In the setting of Proposition 5, the Democratic Party will obtain on average exactly $50 \%$ of seats (independently of voters' attitude towards risk), and the expected policy implemented by the parliament will be

$$
x^{*}=\frac{d^{*}+r^{*}}{2}=\frac{1}{2}-\frac{\tau}{\bar{\pi}_{I}}\left(\bar{\pi}_{D}-\bar{\pi}_{R}\right)-\frac{2 a \tau}{b \bar{\pi}_{I}}
$$

Proof. Plug (A.6)-(A.7) into (A.5), and obtain $S^{*}=1 / 2$, and $x^{*}$ as in (A.9).

We then plug in (A.9) into the social welfare function as in (10). The social planner will decide the seat-vote curve (i.e. $a$ and $b$ ) such that social welfare is maximized.

\footnotetext{
${ }^{13}$ We performed numerical simulations with linear seat-vote curve and higher-degree utility functions (up to the fifth power) and also assuming a log-odds functional form for the seat-vote curve as in

$$
\log \left(\frac{S}{1-S}\right)=a+b \log \left(\frac{V}{1-V}\right)
$$

obtaining the same qualitative results as in the case analyzed in this section regarding the direction of the seat-vote curve bias, i.e. a consistent (weakly) counterbalancing bias. The results are available on request.
} 
Proposition 6. If prefences are linear, the social planner should implement a seat-vote curve such that $a^{*}=0$, while the optimal value of the responsiveness $b$ is indeterminate. If voters' utilities are concave, the optimal bias and responsiveness must be such that

$$
a^{*}=-\frac{\left(\bar{\pi}_{D}-\bar{\pi}_{R}\right)\left(2 \tau-\bar{\pi}_{I}\right)}{4 \tau} \cdot b^{*}
$$

Proof. The social welfare function can be written as:

$$
\begin{array}{r}
W(a, b, \rho=1)=-\bar{\pi}_{D} x^{*}-\bar{\pi}_{R}\left(1-x^{*}\right)+ \\
-\frac{\bar{\pi}_{I}}{4 \epsilon \tau}\left\{\int_{\frac{1}{2}-\epsilon}^{\frac{1}{2}+\epsilon} \int_{m-\tau}^{x^{*}}\left(x^{*}-y\right) d y+\int_{x^{*}}^{m+\tau}\left(y-x^{*}\right) d y d m\right\}, \\
W(a, b, \rho=2)=-\bar{\pi}_{D} x^{* 2}-\bar{\pi}_{R}\left(1-x^{*}\right)^{2}-\frac{\bar{\pi}_{I}}{4 \epsilon \tau} \int_{\frac{1}{2}-\epsilon}^{\frac{1}{2}+\epsilon} \int_{m-\tau}^{m+\tau}\left(x^{*}-y\right)^{2} d y d m
\end{array}
$$

with $x^{*}$ as in (A.9). First-order conditions are necessary and sufficient.

$$
\begin{gathered}
\text { If } \rho=1,\left\{\begin{array}{l}
\frac{\partial W}{\partial a}=0 \text { for } a=0 \\
\left.\frac{\partial W}{\partial b}\right|_{a=0} \equiv 0 \rightarrow b \text { is indeterminate }
\end{array}\right. \\
\text { If } \rho=2, \underset{a}{\arg \max } W=a^{*}=-\frac{\left(\bar{\pi}_{D}-\bar{\pi}_{R}\right)\left(2 \tau-\bar{\pi}_{I}\right)}{4 \tau} \cdot b^{*}
\end{gathered}
$$

Comparing Proposition 4 and Conjecture 2 with Proposition 6, it is clear that our findings hold also when we assume a different decision-making process in the legislature also when legislative bargaining is used. ${ }^{14}$

\section{Proof of Lemma 1}

As from expression (5), the Democratic Party carries the median district and therefore wins the elections if:

$$
\kappa_{M}>-\frac{\frac{r+d}{2}-m}{\tau} \text {, i.e. } m<\frac{r+d}{2}+\tau \kappa_{M}
$$

As $m \sim U\left[\frac{1}{2}-\epsilon, \frac{1}{2}+\epsilon\right]: p=H\left(\frac{r+d}{2}+\tau \kappa_{M}\right)$, i.e. $\quad p=\frac{1}{2}+\frac{r+d}{4 \epsilon}-\frac{1}{4 \epsilon}+\frac{\tau \kappa_{M}}{2 \epsilon}$

\footnotetext{
${ }^{14}$ The bias of the optimal seat-vote curve in the case of non-linear preferences is negative as long as $\bar{\pi}_{I}<2 \tau$. This is always the case according to the empirical simulations reported in the next section.
} 


\section{Proof of Proposition 1}

Each party's best-response function can be found through the first order condition of their respective expected utility:

$$
E\left[U^{D}\right]=p[\Omega-d]-(1-p) r, \quad E\left[U^{R}\right]=p(d-1)+(1-p)[\Omega+r-1]
$$

with $p$ as in Lemma 1. From these we can derive the first-order conditions:

$$
\frac{\partial E\left[U^{D}\right]}{\partial d}: \frac{1-2 \epsilon-2 \tau \kappa-2 d+\Omega}{4 \epsilon}=0, \frac{\partial E\left[U^{R}\right]}{\partial r}: \frac{-1+2 \epsilon-2 \tau \kappa+2(1-r)-\Omega}{4 \epsilon}=0
$$

These are the (dominant-strategy) party best-responde function. Solving for $d$ and $r$, one obtains the policy platforms as in Proposition 1.

\section{Proof of Propositions 2}

$$
\begin{gathered}
d^{*} \in[\bar{m}-\tau, 1 / 2+\tau-\epsilon], \text { and } r^{*} \in[1 / 2-\tau+\epsilon, \bar{m}+\tau] \text { with } \bar{m} \text { as in (9) implies } \\
x \in[\tilde{m}-\tau, \tilde{m}-\tau], \forall \tilde{m} \in\left[\frac{1}{2}-\epsilon, \frac{1}{2}+\epsilon\right], \text { with } x \in\left\{d^{*}, r^{*}\right\}
\end{gathered}
$$

i.e. that for any realization of $\tilde{m}$, there is always a mass of Independents to the left and to the right of each of the two policy platforms $\left\{d^{*}, r^{*}\right\}$. We can therefore re-write the social welfare function as in (10) getting rid of the absolute-value operator, and considering the result showed in Lemma 1, and Proposition 1:

$$
\begin{array}{r}
W\left(\kappa_{M}\right)=-\bar{\pi}_{D} \frac{d^{*}+r^{*}}{2}-\bar{\pi}_{R} \frac{\left(1-d^{*}\right)+\left(1-r^{*}\right)}{2}+ \\
+\bar{\pi}_{I} \int_{\frac{1}{2}-\epsilon}^{\bar{m}}\left[\int_{m-\tau}^{d^{*}}\left[-\left(d^{*}-x\right)\right] f(x) d x+\int_{d^{*}}^{m+\tau}\left[-\left(x-d^{*}\right)\right] f(x) d x\right] h(m) d m+ \\
+\bar{\pi}_{I} \int_{\bar{m}}^{\frac{1}{2}+\epsilon}\left[\int_{m-\tau}^{r^{*}}\left[-\left(r^{*}-x\right)\right] f(x) d x+\int_{r^{*}}^{m+\tau}\left[-\left(x-r^{*}\right)\right] f(x) d x\right] h(m) d m
\end{array}
$$

which takes into account how the social planner does not directly choose the welfare maximising policy, but rather maximizes the sum of the expected utility of each voter, taking into consideration the effect of his or her choice of districting on parties' endogenous behavior.

Knowing $\left\{d^{*}, r^{*}\right\}$ from Proposition 1, we derive the first- and second-order conditions:

$$
\frac{\partial W}{\partial \kappa_{M}}: \tau\left(\bar{\pi}_{D}-\bar{\pi}_{R}-\bar{\pi}_{I} \kappa_{M}\right)=0, \quad \frac{\partial^{2} W}{\partial \kappa_{M}^{2}}=-\tau \bar{\pi}_{I}
$$

Solving the (necessary and sufficient) first-order condition for $\kappa_{M}$ we obtain:

$$
\kappa_{M}^{*} \equiv \underset{\kappa_{M}}{\arg \max } W=\frac{\bar{\pi}_{D}-\bar{\pi}_{R}}{\bar{\pi}_{I}}
$$


From these results, simple algebra shows us that in equilibrium $d^{*}\left(\kappa_{M}^{*}\right)$ and $r^{*}\left(\kappa_{M}^{*}\right)$ belong to the intervals as in the first line of this proof as long as $(\bar{\kappa} \in[a, b])$.

In the cases when $\bar{\kappa} \notin[a, b]$, the social welfare function is no longer equal to the one in (A.10), as for some realizations of $\tilde{m}$ all the Independent voters lie on the same side of the winning policy.

As an example, here is the proof for one of the cases one could analyze:

Special Case: $\bar{\kappa} \in[b, 1], \bar{\pi}_{D}>\bar{\pi}_{R}$

If $\bar{\kappa} \in[b, 1]$, simple algebra shows us that $d^{*}<\bar{m}-\tau$. The welfare of partisan voters (as in the first line of [A.10]) in unchanged, while the Independent voters' welfare WInd' becomes

$$
\begin{aligned}
W \operatorname{Ind}\left(\kappa_{M}\right)^{\prime}= & \int_{\frac{1}{2}-\epsilon}^{d^{*}+\tau}\left[\int_{m-\tau}^{d^{*}}\left[-\left(d^{*}-x\right)\right] f(x) d x+\int_{d^{*}}^{m+\tau}\left[-\left(x-d^{*}\right)\right] f(x) d x\right] h(m) d m+ \\
& +\int_{d^{*}+\tau}^{\bar{m}}\left[\int_{m-\tau}^{m+\tau}\left[-\left(x-d^{*}\right)\right] f(x) d x\right] h(m) d m+ \\
& +\int_{\bar{m}}^{\frac{1}{2}+\epsilon}\left[+\int_{m-\tau}^{r^{*}}\left[-\left(r^{*}-x\right)\right] f(x) d x+\int_{r^{*}}^{m+\tau}\left[-\left(x-r^{*}\right)\right] f(x) d x\right] h(m) d m
\end{aligned}
$$

Note the difference between the second line of (A.10) and the first two of (A.11). Concavity is preserved, but for some realization of $\tilde{m}$ all Independents lie to the right of $d^{*}$, breaking the "symmetry" of (A.10).

We need to show that

$$
\underset{\kappa_{M}}{\arg \max } W \leq \underset{\kappa_{M}}{\arg \max } W^{\prime}
$$

The two welfare functions are twice continuously differentiable, strictly concave in $\kappa_{M}$. They are in fact simple parabolas. As

$$
\left\{\frac{\partial W}{\partial \kappa_{M}}<0 \text { for } \kappa_{M}>\underset{\kappa_{M}}{\arg \max } W\right\} \text { and }\left\{\frac{\partial W^{\prime}}{\partial \kappa_{M}}>0, \text { for } \kappa_{M}<\underset{\kappa_{M}}{\arg \max } W^{\prime}\right\}
$$

Then

$$
\tilde{\kappa}_{M} \equiv\left\{\kappa_{M} \mid \frac{\partial W}{\kappa_{M}}-\frac{\partial W^{\prime}}{\kappa_{M}}=0\right\} \Rightarrow \underset{\kappa_{M}}{\arg \max } W \leq \tilde{\kappa}_{M} \leq \underset{\kappa_{M}}{\arg \max } W^{\prime}
$$

It can be calculated that $\tilde{\kappa}_{M}=1+(\Omega-2 \epsilon) /(2 \tau)$, therefore we need to show that

$$
1+\frac{\Omega-2 \epsilon}{2 \tau} \geq \frac{\bar{\pi}_{D}-\bar{\pi}_{R}}{\bar{\pi}_{I}}
$$

Considering that we are analyzing the case in which $\frac{\bar{\pi}_{D}-\bar{\pi}_{R}}{\bar{\pi}_{I}} \in[b, 1],(\mathrm{A} .14)$ can be rewritten 
as

$$
1+\frac{\Omega-2 \epsilon}{2 \tau} \geq \min \left[1-\frac{\Omega}{2 \tau}, 1+\frac{\Omega-2 \epsilon}{2 \tau}\right]
$$

which is always true.

\section{Proof of Proposition 3}

The welfare function as in (10) can be re-written as:

$$
\begin{gathered}
W\left(\kappa_{M}, \rho\right)=\bar{\pi}_{D}[p L(0, d)+(1-p) L(0, r)]+\bar{\pi}_{R}[p L(1, d)+(1-p) L(1, r)]+ \\
+\bar{\pi}_{I} \int_{\frac{1}{2}-\epsilon}^{\bar{m}} \int_{m-\tau}^{m+\tau} L\left(x, d^{*}\right) f(x) d x h(m) d m+\bar{\pi}_{I} \int_{\bar{m}}^{\frac{1}{2}+\epsilon} \int_{m-\tau}^{m+\tau} L\left(x, r^{*}\right) f(x) d x h(m) d m
\end{gathered}
$$

with $p$ as in Lemma 1 , and $\left\{d^{*}, r^{*}\right\}$-and consequently also $\{p, \bar{m}\}$-depending on both $\rho$ and $\kappa_{M}$. For simplicity of explanation we can re-define the welfare function as:

$$
W=\bar{\pi}_{D} W^{D e m}+\bar{\pi}_{R} W^{R e p}+\bar{\pi}_{I} W^{I n d}
$$

Subscripts will refer to partial differentials. We need to demonstrate that: $\mathrm{d} \kappa_{M}^{*} /\left.\mathrm{d} \rho\right|_{\rho=1}<0$, which by the Implicit Function Theorem is equivalent to:

$$
\begin{array}{r}
-\left.\frac{W_{\kappa_{M}, \rho}}{W_{\kappa_{M}, \kappa_{M}}}\right|_{\kappa_{M}^{*}, \rho=1}<0, \text { with } \kappa_{M}^{*}(\rho=1)={ }^{\left(\bar{\pi}_{D}-\bar{\pi}_{R}\right) / \bar{\pi}_{I}} \\
\text { Moreover: } W_{\kappa_{M}, \kappa_{M}}\left(\kappa_{M}^{*}, \rho=1\right)=-\tau \bar{\pi}_{I} \Rightarrow \\
\Rightarrow \operatorname{signum}\left\{-\left.\frac{W_{\kappa_{M}, \rho}}{W_{\kappa_{M}, \kappa_{M}}}\right|_{\kappa_{M}^{*}, \rho=1}\right\} \equiv \operatorname{signum}\left\{W_{\kappa_{M}, \kappa_{M}}\left(\kappa_{M}^{*}, \rho=1\right)\right\}
\end{array}
$$

It can be said that:

$$
W_{\rho, \kappa_{M}}=\frac{\partial^{2} W}{\partial \rho \partial \kappa_{M}}+W_{d, \rho} \frac{\mathrm{d} d^{*}}{\mathrm{~d} \kappa_{M}}+W_{r, \rho} \frac{\mathrm{d} r^{*}}{\mathrm{~d} \kappa_{M}}+W_{d} \frac{\mathrm{d}^{2} d^{*}}{\mathrm{~d} \rho \kappa_{M}}+W_{r} \frac{\mathrm{d}^{2} r^{*}}{\mathrm{~d} \rho \kappa_{M}}
$$

From Proposition 1 we know that $\left.\frac{\mathrm{d} d^{*}}{\mathrm{~d} \kappa_{M}}\right|_{\rho=1}=\left.\frac{\mathrm{d} r^{*}}{\mathrm{~d} \kappa_{M}}\right|_{\rho=1}=-\tau$. Using the implicit function theorem, we can find $\left\{\mathrm{d} d^{*} / \mathrm{d} \rho, \mathrm{d}^{*} / \mathrm{d} \rho\right\}$, and carefully differentiating with respect to $\kappa_{M}$ :

$$
\begin{array}{r}
\left.\frac{\mathrm{d}^{2} d^{*}}{\mathrm{~d} \rho \mathrm{d} \kappa_{M}}\right|_{\kappa_{M}^{*}, \rho=1}=\frac{\tau}{2}\left(\log d^{*}-\log r^{*}\right)+\frac{\epsilon \tau}{d^{*}} \\
\left.\frac{\mathrm{d}^{2} r^{*}}{\mathrm{~d} \rho \mathrm{d} \kappa_{M}}\right|_{\kappa_{M}^{*}, \rho=1}=\frac{\tau}{2}\left[\log \left(1-r^{*}\right)-\log \left(1-d^{*}\right)\right]+\frac{\epsilon \tau}{1-r^{*}}
\end{array}
$$

It can be calculated that $W_{d}^{\text {Dem }}=W_{r}^{\text {Dem }}=W_{d}^{R e p}=W_{r}^{\text {Rep }}=0$, and that $W_{d, \rho}^{\text {Ind }}=W_{r, \rho}^{\text {Ind }}=$ 
0. Therefore:

$$
\begin{array}{r}
W_{\kappa_{M}, \rho}^{D e m}=\tau \bar{\pi}_{D}\left[\log \left(d^{*}\right)+\log \left(r^{*}\right)+2\right] / 2 \\
W_{\kappa_{M}, \rho}^{R e p}=-\tau \bar{\pi}_{R}\left[\log \left(1-d^{*}\right)+\log \left(1-r^{*}\right)+2\right] / 2 \\
W_{\kappa_{M}, \rho}^{I n d}=\bar{\pi}_{I}\left\{\tau \kappa_{M} / 2+\left[f\left(\tau-\tau \kappa_{M}+\epsilon\right)+f\left(\tau+\tau \kappa_{M}-\epsilon\right)+\right.\right. \\
\left.\left.-f\left(\tau-\tau \kappa_{M}-\epsilon\right)-f\left(\tau+\tau \kappa_{M}+\epsilon\right)\right] /(8 \epsilon)\right\} \\
\text { with } f(x)=x^{2} \log (x)
\end{array}
$$

$W_{\kappa_{M}, \rho}$ will be evaluated at $\left\{\kappa_{M}^{*}=\left(\bar{\pi}_{D}-\bar{\pi}_{R}\right) / \bar{\pi}_{I}, \rho=1\right\}$, and its behavior will be analyzed, in order to prove that is always negative for any value of the relevant parameter. In particular its behavior w.r.t. $\bar{\pi}_{D}$, holding everything else constant (i.e. for any feasible value of the other parameters).

Step 1 It can be easily calculated that $\left.\left\{W_{\rho, \kappa_{M}}^{\text {Dem }}+W_{\rho, \kappa_{M}}^{R e p}\right\}\right|_{\kappa_{M}=0}=\left.\left\{W^{I n d}\right\}\right|_{\kappa_{M}=0}=$ 0 .

Step $2 W^{D R}=\left(W^{D e m}+W^{R e p}\right)$ decreases in $\bar{\pi}_{D}$

$$
\left.\frac{\partial W^{D R}\left(\kappa_{M}^{*}\right)}{\partial \bar{\pi}_{D}}\right|_{\kappa_{M}^{*}}=\overbrace{W_{\kappa_{M}}^{D e m}}^{-}+\underbrace{\left(\bar{\pi}_{D}-\bar{\pi}_{R}\right)}_{+} \overbrace{W_{\kappa_{M}}^{D e m}}^{-} \underbrace{\kappa_{M}^{\prime}}_{+}+\bar{\pi}_{R} \overbrace{W_{\kappa_{M}}^{D R}}^{-} \underbrace{\kappa_{M}^{\prime}}_{+}<0
$$

where "primes" on $\kappa_{M}$ indicate partial derivatives of $\kappa_{M}^{*}$ w.r.t. $\bar{\pi}_{D}$. As:

$$
W_{\kappa_{M}}^{D e m}=-\tau^{2}\left(d^{-1}+r^{-1}\right) / 2, \quad W_{\kappa_{M}}^{D R}=-\tau^{2}\left[d^{-1}+r^{-1}+(1-d)^{-1}+(1-r)^{-1}\right] / 2
$$

Step $3 W^{D R}=\left(W^{D e m}+W^{R e p}\right)$ is concave in $\bar{\pi}_{D}$

$$
\begin{array}{r}
\left.\frac{\partial^{2} W^{D R}\left(\kappa_{M}^{*}\right)}{\partial \bar{\pi}_{D}^{2}}\right|_{\kappa_{M}^{*}}=2 \overbrace{W_{\kappa_{M}}^{D e m}}^{-} \overbrace{\kappa_{M}^{\prime}}^{+}+\overbrace{\left(\bar{\pi}_{D}-\bar{\pi}_{R}\right)}^{+}[\overbrace{W_{\kappa_{M} \kappa_{M}}^{D e m}}^{-} \overbrace{\kappa_{M}^{\prime}}^{+}+\overbrace{W_{\kappa_{M}}^{D e m}}^{-} \overbrace{\kappa_{M}^{\prime \prime}}^{+}]+ \\
+\bar{\pi}_{R}[\underbrace{W_{\kappa_{M} \kappa_{M}}^{D R}}_{-}\left(\kappa_{M}^{\prime}\right)^{2}+\underbrace{W_{\kappa_{M}}^{D R}}_{-} \underbrace{\kappa_{M}^{\prime \prime}}_{+}]<0
\end{array}
$$

As $\quad W_{\kappa_{M} \kappa_{M}}^{D R}=\tau^{3}\left[-d^{-2}-r^{-2}+(1-d)^{-2}+(1-r)^{-2}\right] / 2<0$

Step $4 W^{I n d}$ is convex in $\bar{\pi}_{D}$.

It can be calculated that $\left.\frac{\partial^{2} W^{I n d}\left(\kappa_{M}^{*}\right)}{\partial \bar{\pi}_{D}^{2}}\right|_{\bar{\pi}_{D}=\bar{\pi}_{R}}=0$, moreover 


$$
\frac{\partial^{3} W^{I n d}\left(\kappa_{M}^{*}\right)}{\partial \bar{\pi}_{D}^{3}}=W I^{\prime \prime}\left[-3\left(\kappa_{M}^{\prime}\right)^{2}+3 \bar{\pi}_{I} \kappa_{M}^{\prime} \kappa_{M}^{\prime \prime}\right]+\bar{\pi}_{I} W I^{\prime \prime \prime}\left(\kappa_{M}^{\prime}\right)^{3}>0
$$

where $W I=W^{I n d} / \bar{\pi}_{I}$ and the "primes" next to $W I$ indicate partial derivatives w.r.t. $\kappa_{M}$. As:

$$
\begin{array}{r}
W I^{\prime \prime \prime}=\frac{\tau^{3}\left(\tau^{2}+\tau^{2} \kappa_{M}^{2}-\epsilon^{2}\right)}{\left(\tau-\tau \kappa_{M}+\epsilon\right)\left(\tau+\tau \kappa_{M}-\epsilon\right)\left(\tau-\tau \kappa_{M}-\epsilon\right)\left(\tau+\tau \kappa_{M}+\epsilon\right)}>0 \\
W I^{\prime \prime}=\frac{\epsilon}{8} \log \left(\frac{\tau^{2}-\left(\tau \kappa_{M}-\epsilon\right)^{2}}{\tau^{2}-\left(\tau \kappa_{M}+\epsilon\right)^{2}}\right)>0,-3\left(\kappa_{M}^{\prime}\right)^{2}+3 \bar{\pi}_{I} \kappa_{M}^{\prime} \kappa_{M}^{\prime \prime}=\frac{3\left(1-2 \bar{\pi}_{R}\right)^{2}}{\bar{\pi}_{I}^{4}}>0
\end{array}
$$

$W^{\text {Ind }}$ 's second derivative is zero at $\kappa_{M}=0$, while the third derivative is always positive. This implies that $W^{I n d}$ is convex, and therefore that its maximum is at either of its extremes. As $W^{D R}=W^{I n d}\left(\kappa_{M}=0\right)=0$, and as $W^{D R}$ is concave and decreasing at $\kappa_{M}=0$, a sufficient condition for $W_{\kappa_{M}^{*}, \rho=1}$ to be non-positive is to consider the other local maximum of $W^{I n d}$, i.e. when $\kappa_{M}=1-\epsilon / \tau$, and when $\bar{\pi}_{I}$ is at its maximum given the parameter ranges and the value of $\kappa_{M}$. This is at $\epsilon \rightarrow 0, \tau \rightarrow 1 / 4$, which implies $\kappa_{M}^{\max }=1$. At these values

$$
\left\{W^{\text {Dem }} / \bar{\pi}_{D}=.25-.5 \log 2, W^{R e p} / \bar{\pi}_{R}=-.25(1+\log 3-\log 4), W I=.25 \log 2\right\}
$$

This implies a negative value of $W_{\kappa_{M}, \rho=1}<0$, for any value of $\left\{\bar{\pi}_{D}, \bar{\pi}_{R}\right\}$ s.t. $\kappa_{M}^{*}=1$.

\section{Proof of Proposition 4}

Let's re-define in a more convenient way the skewness of the socially optimal median district:

$$
\kappa_{M}^{*}=\frac{\bar{\pi}_{D}-\bar{\pi}_{R}}{\bar{\pi}_{I}}+\lambda
$$

The vote share of the Democratic party at the national level $V(m)$ and in the median district $V_{M}(m)$ are:

$$
V(m)=\bar{\pi}_{D}+\frac{\bar{\pi}_{I}}{2 \tau}\left(\frac{r+d}{2}-m+\tau\right), V_{M}(m)=\pi_{D M}+\frac{\pi_{I M}}{2 \tau}\left(\frac{r+d}{2}-m+\tau\right)
$$

From (A.17), we can say that the median district is carried by the Democratic party if:

$$
\tilde{m}<\frac{d+r}{2}+\tau \kappa_{M} \Rightarrow V_{M}(\tilde{m})>\frac{1}{2}
$$

From (A.17) we can also find out when the Democratic party obtains more than $50 \%$ of the votes nationally if:

$$
\tilde{\tilde{m}}<\frac{d+r}{2}+\tau\left(\frac{\bar{\pi}_{D}-\bar{\pi}_{R}}{\bar{\pi}_{I}}\right) \Rightarrow V(\tilde{\tilde{m}})>\frac{1}{2}
$$


Therefore if the districting reflect the population composition (as in Proposition 2 and $(\bar{\kappa} \in[a, b]))$, i.e.

$$
\text { If } \kappa_{M}^{*}=\frac{\bar{\pi}_{D}-\bar{\pi}_{R}}{\bar{\pi}_{I}} \text {, then } V_{M}=\frac{1}{2} \Rightarrow V=\frac{1}{2}
$$

which implies that when the median district exactly reflects the population composition the seat-vote curve bias is zero, as stated in Proposition 4, taking into consideration the results of Proposition 2.

If the districting is "more extremist" than the population as a whole, then, substituting into (A.17),

$$
\tilde{m}=\frac{d+r}{2}+\tau\left(\frac{\bar{\pi}_{D}-\bar{\pi}_{R}}{\bar{\pi}_{I}}\right) \Rightarrow V=\frac{1}{2} \text { and }\left\{\begin{array}{l}
V_{M}>\frac{1}{2} \text { if } \bar{\pi}_{D}>\bar{\pi}_{R} \\
V_{M}<\frac{1}{2} \text { if } \bar{\pi}_{D}<\bar{\pi}_{R}
\end{array}\right.
$$

i.e. a majority of seats (i.e. carrying the median district) with $50 \%$ of votes for the majoritarian party implies a reinforcing bias, as stated in Lemma 2.

If the districting is "more centrist" as the population composition, then, substituting into (A.17),

$$
\tilde{m}=\frac{d+r}{2}+\tau\left(\frac{\bar{\pi}_{D}-\bar{\pi}_{R}}{\bar{\pi}_{I}}\right) \Rightarrow V=\frac{1}{2} \text { and }\left\{\begin{array}{l}
V_{M}<\frac{1}{2} \text { if } \bar{\pi}_{D}>\bar{\pi}_{R} \\
V_{M}>\frac{1}{2} \text { if } \bar{\pi}_{D}<\bar{\pi}_{R}
\end{array}\right.
$$

A minority of seats (i.e. not carrying the median district) with $50 \%$ of votes for the majoritarian party implies a counterbalancing bias, as stated in Propositions 4 and Conjecture 2 .

\section{Proof of Lemma 2}

From Proposition 4 and its proof, we know that a median district, which is "more centrist" than the whole population implies a "counterbalancing" bias. From Proposition 3 we know that in a neighborhood of the linear equilibrium, the socially optimal median district becomes more centrist as voters' utility becomes more concave.

\section{Feasibility: an Intuitive Argument}

Districts with the same median-district partisan slant $\kappa_{M}$ are equivalent from the equilibrium and welfare point of view. Consequently many different districting plans fulfil a given optimality condition.

Let's focus on a special simple case in which there is a very large number of districts, all districts to the left (right) of the median have the same composition, and Independents are uniformly distributed across districts, i.e.:

Assumption 3. $\kappa_{j}=c \quad \forall j<M ; \quad \kappa_{j}=C \quad \forall j>M ; \quad \pi_{I j}=\bar{\pi}_{I} \quad \forall j$

Obviously, just by the very definition of median, we see true that $\kappa_{c} \leq \kappa_{M} \leq \kappa_{C}$.

Let's focus first on the case $\bar{\pi}_{D} \geq \bar{\pi}_{R}$, and then generalize our results. Let us find out which is the "most extreme" districting that is feasible under these assumptions (so 
as to maximize $\left(\kappa_{C}-\kappa_{c}\right)$ ), in order to find the span within which the social planner can design the median district $\kappa_{M}$. Roughly speaking, we are moving all Republican voters to districts on the left-hand side of the median one (substituting them with Democratic voters who belonged to districts on the left-hand side). ${ }^{15}$ The resulting districting and partisan slants are the following:

$$
\begin{array}{r}
\pi_{D c}=\bar{\pi}_{D}-\bar{\pi}_{R}, \pi_{R c}=2 \bar{\pi}_{R} ; \pi_{D C}=\bar{\pi}_{D}+\bar{\pi}_{R} ; \quad \pi_{R C}=\bar{\pi}_{R}-\bar{\pi}_{R}=0 ; \\
\pi_{I c}=\pi_{I C}=\pi_{I M}=\bar{\pi}_{I} \\
\kappa_{c}=\frac{\bar{\pi}_{D}-3 \bar{\pi}_{R}}{\bar{\pi}_{I}}, \quad \kappa_{C}=\frac{\bar{\pi}_{D}+\bar{\pi}_{R}}{\bar{\pi}_{I}}, \quad \kappa_{M} \in\left[\kappa_{c}, \kappa_{C}\right]
\end{array}
$$

More generally, considering also the case in which $\bar{\pi}_{D} \leq \bar{\pi}_{R}$, the most extremist feasible districting is such that

$$
\begin{gathered}
\kappa_{M} \in\left[\frac{\bar{\pi}_{D}-3 \bar{\pi}_{R}}{\bar{\pi}_{I}}, \frac{\bar{\pi}_{D}+\bar{\pi}_{R}}{\bar{\pi}_{I}}\right] \text { if } \quad \bar{\pi}_{D} \geq \bar{\pi}_{R} ; \\
\kappa_{M} \in\left[-\frac{\bar{\pi}_{D}+\bar{\pi}_{R}}{\bar{\pi}_{I}}, \frac{3 \bar{\pi}_{D}-\bar{\pi}_{R}}{\bar{\pi}_{I}}\right] \text { if } \quad \bar{\pi}_{D}<\bar{\pi}_{R}
\end{gathered}
$$

Applying these formulae to the NYT-CBS poll data as in Table III, we can see how the feasible interval for $\kappa_{M}$ is always a superset of the interval $[-1,1]$, with only one exception. This simple exercise shows (almost) any median district Democratic slant within $[-1,1]$ can be designed by the districting authority.

\footnotetext{
${ }^{15}$ Remember that for our notation districts are ordered by $\kappa$, therefore go from the most conservative $(\kappa<0)$ to the most liberal $(\kappa>0)$.
} 
Alesina, A., 1988. Credibility and policy convergence in a two-party system with rational voters. The American Economic Review 78 (4), 796-805.

Ansolabehere, S., Snyder, Jr., J. M., 2002. The incumbency advantage in u.s. elections: An analysis of state and federal offices: 1942-2000. Election Law Journal 1, 315-338.

Bernhardt, D., Duggan, J., Squintani, F., 2009. The case for responsible parties. American Political Science Review 103 (4), 570-587.

Besley, T., Coate, S., feb 1997. An economic model of representative democracy. The Quarterly Journal of Economics 112 (1), 85-114.

Besley, T., Preston, I., 2007. Electoral bias and policy choice: Theory and evidence. Quarterly Journal of Economics 122 (4), 1473-1510.

Callander, S., 2008. Political motivations. Review of Economic Studies 75, 671-697.

Calvert, R. L., 1985. Robustness of the multidimensional voting model: Candidate motivations, uncertainty, and convergence. American Journal of Political Science 29 (1), 69-95.

Coate, S., 2004. Pareto-improving campaign finance policy. The American Economic Review 94 (3), 628-655.

Coate, S., Knight, B., 2007. Socially optimal districting: A theoretical and empirical exploration. Quarterly Journal of Economics 122 (4), 1409-1471.

Downs, A., 1957. An economic theory of democracy. Harper and Row, New York.

Epstein, D., O'Hallaran, S., 2004. The 45\% solution: Racial gerrymandering and representative democracy. Working paper, Columbia University.

Gelman, A., King, G., 1994. A unified method of evaluating electoral systems and redistricting plans. American Journal of Political Science 38 (2), 514-554.

Gilligan, T. W., Matsusaka, J. G., 2006. Public choice principles of redistricting. Public Choice 129, 381-398.

Gul, F., Pesendorfer, W., September 2010. Strategic redistricting. American Economic Review 100 (4), 1616-41.

Handley, L., 2008. A Comparative Survery of Structures and Criteria for Boundary Delimitation. New York: Oxford, pp. 265-83.

Hinich, M. J., Ordeshook, P. C., 1974. The electoral college: A spatial analysis. Political Methodology 1, 1-29.

Kendall, M. G., Stewart, A., 1950. The law of the cubic proportion in election results. British Journal of Sociology 1, 183-196. 
Lizzeri, F., Persico, N., 2001. The provision of public goods under alternative electoral incentives. American Economic Review 91, 225-339.

Lockwood, B., 2002. Distributive politics and the costs of centralization. Review of Economic Studies 69, 313-337.

Osborne, M. J., Slivinski, A., February 1996. A model of political competition with citizencandidates. The Quarterly Journal of Economics 111 (1), 65-96.

Persson, T., Roland, G., Tabellini, G., 2000. Comparative politics and public finance. Journal of Political Economy 108, 1121-1161.

Persson, T., Tabellini, G., 2002. Political economics: explaining economic policy. Zeuthen lecture book series. MIT Press.

Prat, A., 2002. Campaign advertising and voter welfare. Review of Economic Studies 69, 999-1018.

Puppe, C., Tasndi, A., October 2009. Optimal redistricting under geographical constraints: Why "pack and crack" does not work. Economics Letters 105 (1), 93-96.

Sherstyuk, K., 1998. How to gerrymander: A formal analysis. Public Choice 95, 27-49.

Shotts, K. W., 2002. Gerrymandering, legislative composition, and national policy outcomes. American Journal of Political Science 46 (2), 398-414.

Wittman, D., 1983. Candidate motivation: A synthesis of alternative theories. The American Political Science Review 77 (1), 142-157. 